\title{
Direct Evidence for the Participation of Oxygen Vacancies in the Oxidation of Carbon Monoxide over Ceria- Supported Gold Catalysts by using Operando Raman Spectroscopy
}

\author{
Marno Lohrenscheit and Christian Hess ${ }^{*[a]}$
}

\begin{abstract}
Supported gold catalysts are highly active for a variety of reactions, including low-temperature CO oxidation. It has been shown that reducible support materials, for example, ceria and titania, may significantly alter catalytic performance. However, the current understanding of the $\mathrm{CO}$ oxidation mechanism of such gold catalysts is still incomplete, as important details such as the activation of oxygen and the role of oxygen vacancies are unknown. To elucidate the role of the ceria support during room-temperature CO oxidation, we employed operando Raman spectroscopy by simultaneously recording the Raman spectra of the catalyst and the gas-phase FTIR spectra. Our results give first direct spectroscopic evidence for the participation of oxygen vacancies in the oxidation of $\mathrm{CO}$ over ceria-supported gold, which thus underlines the crucial role of the support material for detailed understanding of the mode of operation of supported gold catalysts.
\end{abstract}

Nanosized gold particles dispersed on metal oxide supports are highly active for various reactions such as low-temperature CO oxidation, preferential CO oxidation, and (reverse) water gas shift. ${ }^{[1-6]}$ Although there has been considerable progress in the field, detailed mechanistic understanding of the mode of operation of these catalysts is still missing. It is generally accepted that the gold-support interface perimeter acts as the active site, the oxide support strongly influences the catalytic properties, and the gold particle size plays an important role in catalytic activity. ${ }^{[7-10]}$ Furthermore, it has been shown that the characteristics of reducible support materials such as ceria may significantly alter catalytic performance. ${ }^{[1]}$ Regarding the mechanism of CO oxidation over ceria-supported gold, important details such as the activation of oxygen as well as the role of oxygen vacancies are still unknown. Oxygen activation may proceed without participation of oxygen vacancies, via vacancy-stabilized oxygen intermediates, or by direct participation of lattice oxygen. Previous Raman work focused on $F_{2 g}$ phonon softening and the role of ceria bulk defects. ${ }^{[12]}$ The stabilization of oxygen on one- and two-electron defect sites and the in-

[a] M. Lohrenscheit, Prof. Dr. C. Hess

Eduard-Zintl-Institut für Anorganische und Physikalische Chemie Technische Universität Darmstadt

Alarich-Weiss-Strasse 8, 64287 Darmstadt (Germany)

E-mail: hess@pc.chemie.tu-darmstadt.de

$\square$ Supporting Information for this article is available on the WWW under http://dx.doi.org/10.1002/cctc.201501129. volvement of such species in CO oxidation has been proposed previously on the basis of Raman spectra recorded before and after the reaction. ${ }^{[13]}$ On the basis of theoretical studies on ceria-supported gold nanoparticles, the participation of oxygen vacancies in $\mathrm{CO}$ oxidation has been proposed. ${ }^{[14]}$ In this contribution, we present the first direct spectroscopic evidence for the participation of oxygen vacancies in $\mathrm{CO}$ oxidation over ceria-supported gold catalysts (Au/ceria) by simultaneously recording the Raman spectra of the catalyst and the gas-phase FTIR spectra during the reaction (operando Raman spectroscopy) and relating the dynamic behavior of catalyst surface species with catalytic activity.

Figure 1 depicts the temporal evolution of $\mathrm{CO}$ oxidation activity over $0.5 \mathrm{wt} \% \mathrm{Au} / \mathrm{ceria}$ at $25^{\circ} \mathrm{C}$, as monitored by FTIR gas-phase spectroscopy (for details on sample preparation and characterization, see the Experimental Section and Figure S1 in the Supporting Information). As shown in the top panel of this figure, the gas feed was cycled between $\mathrm{Ar} / \mathrm{O}_{2}(3: 1)$ and $\mathrm{Ar} /$ $\mathrm{CO} / \mathrm{O}_{2}(25: 1: 5)$ at a total flow rate of $100 \mathrm{~mL} \mathrm{~min}{ }^{-1}$. The $\mathrm{CO}$ conversions were determined as 9.8 and $6.1 \%$ at the maximum and end of the first $\mathrm{Ar} / \mathrm{CO} / \mathrm{O}_{2}$ run, respectively. The bottom panel of Figure 1 shows an expanded view of the activity in the first $\mathrm{Ar} / \mathrm{CO} / \mathrm{O}_{2}$ run. A comparison of the gas-phase signals reveals an initially slower response of the $\mathrm{CO}_{2}$ signal, which is indicative of an activation period of the $\mathrm{CO}$ oxidation reaction, in agreement with recent studies. ${ }^{[15]}$ During the course of the reaction, the activity drops slowly after reaching its maximum after approximately $10 \mathrm{~min}$. As a reason for the deactivation, the formation of surface carbonates has been proposed on the basis of diffuse reflectance infrared Fourier transform spectroscopy (DRIFTS). ${ }^{[15]}$

Figure 2 depicts the operando Raman spectra of $0.5 \mathrm{wt} \%$ $\mathrm{Au} /$ ceria taken during switching between non-reactive $\left(\mathrm{Ar} / \mathrm{O}_{2}\right)$ and reactive $\left(\mathrm{Ar} / \mathrm{CO} / \mathrm{O}_{2}\right)$ conditions, as indicated in the top panel of Figure 1. The Raman spectra were recorded with a fiber optic probe by using $\lambda=532 \mathrm{~nm}$ laser excitation (for details on the operando setup, see the Experimental Section). ${ }^{[16]}$ The Raman spectra of $\mathrm{Au} / \mathrm{ceria}$ are dominated by a strong band at a shift of $463 \mathrm{~cm}^{-1}$ resulting from the $F_{2 g}$ mode in cubic fluorite. Smaller bands appear at shifts of $255, \approx 590$ (shoulder), 830 (several contributions), and $1172 \mathrm{~cm}^{-1}$, which can be assigned to bulk ceria, bulk defects in ceria, peroxide, and second-order Raman scattering in ceria, respectively (see Figure S2). ${ }^{[17-19]}$ 

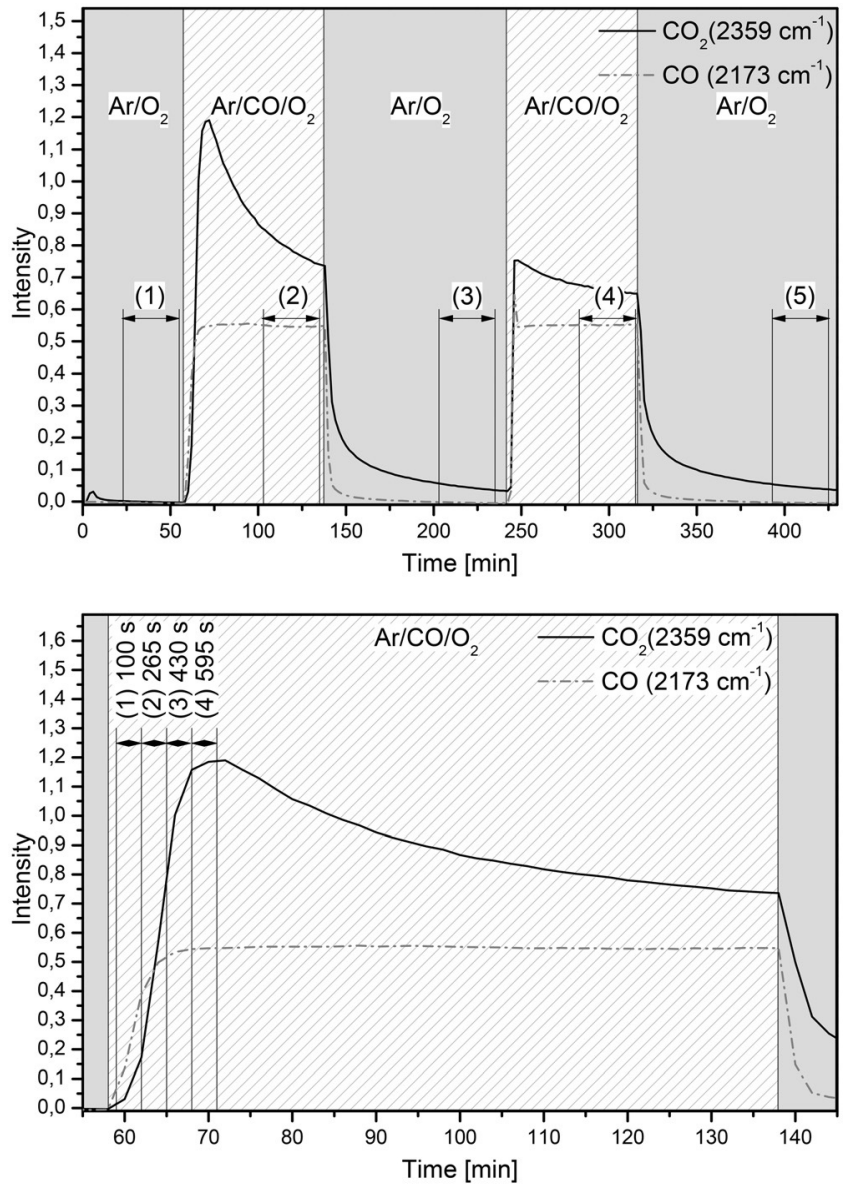

Figure 1. Top) Temporal evolution of the CO oxidation activity over $0.5 \mathrm{wt} \%$ $\mathrm{Au} /$ ceria at $25^{\circ} \mathrm{C}$ as monitored by FTIR gas-phase spectroscopy. The gas feed was cycled between $\mathrm{Ar} / \mathrm{O}_{2}(3: 1)$ and $\mathrm{Ar} / \mathrm{CO} / \mathrm{O}_{2}(25: 1: 5)$ at a total flow rate of $100 \mathrm{~mL} \mathrm{~min}{ }^{-1}$. Bottom) Expanded view of the activity in the first $\mathrm{Ar}$ / $\mathrm{CO} / \mathrm{O}_{2}$ run. Arrows indicate start and end of operando Raman experiments.

The top panel of Figure 2 focuses on spectral changes in the peroxide range. The peroxide band at a shift of approximately $840 \mathrm{~cm}^{-1}$ results from adsorption of molecular oxygen on twoelectron surface-defect sites. ${ }^{[18]}$ Close inspection of this band prior to the reaction [spectrum (1)] shows that the band is composed of (at least) three contributions located at Raman shifts of 832,847 , and $881 \mathrm{~cm}^{-1}$, which were previously assigned to peroxides adsorbed on defect sites with increasing degrees of aggregation. ${ }^{[18]}$ Upon switching from non-reactive to reactive conditions, the intensity of the peroxide band increases [spectrum (2)]. Switching back to oxygen results in a further increase [spectrum (3)], whereas in $\mathrm{Ar} / \mathrm{CO} / \mathrm{O}_{2}$ feed the intensity of the peroxide signal drops back to its original level in $\mathrm{Ar} / \mathrm{CO} / \mathrm{O}_{2}$ [spectrum (4)]. The observed behavior during switching between non-reactive and reactive conditions is summarized in the bottom panel of Figure 2, which shows the difference spectra of the peroxide signal for successive conditions. Whereas the first exposure to reaction conditions leads to a strong increase in peroxide intensity attributed to catalyst rearrangement (e.g., catalyst reduction), subsequent switching results in reversible changes in peroxide intensities with higher overall peroxide concentrations under non-reactive conditions.
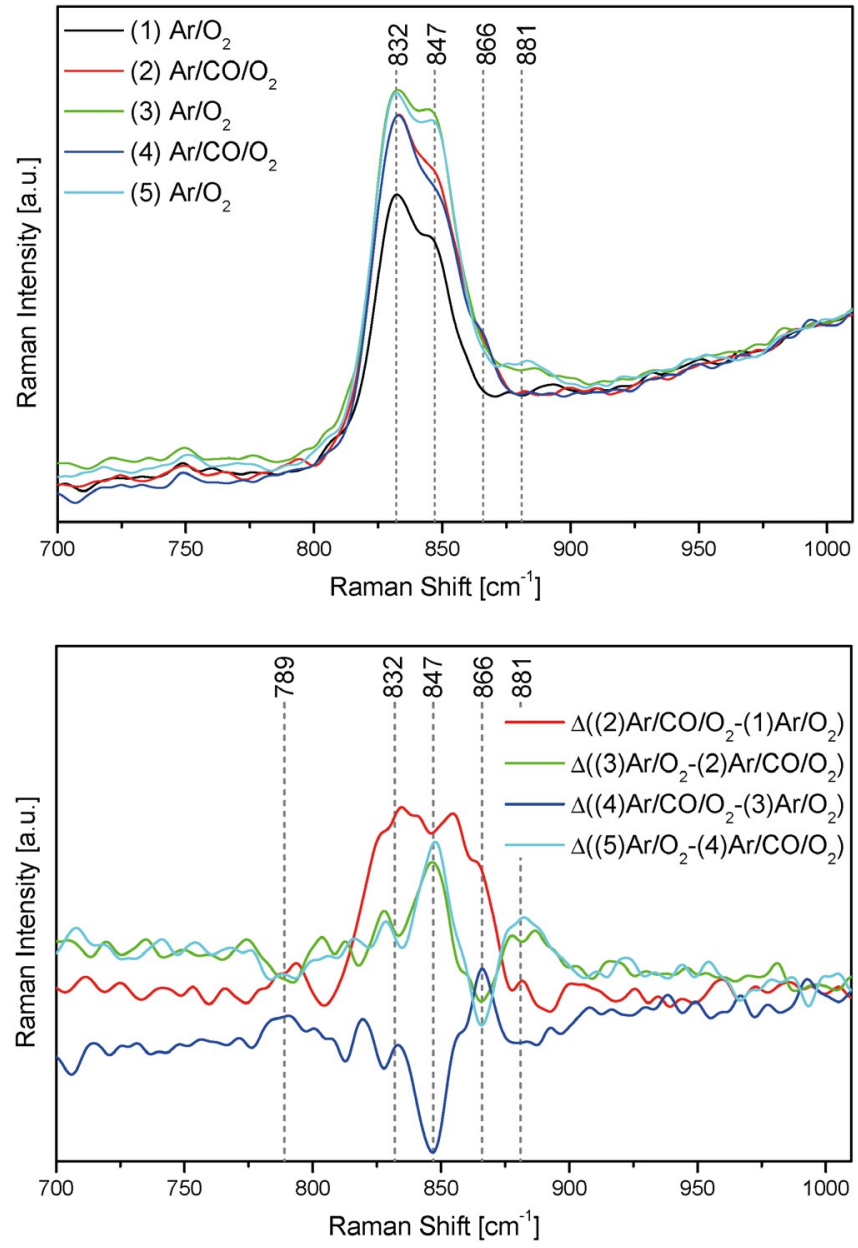

Figure 2. Operando Raman spectra of $0.5 \mathrm{wt} \% \mathrm{Au} /$ ceria taken during cycling between $\mathrm{Ar} / \mathrm{O}_{2}(3: 1)$ and $\mathrm{Ar} / \mathrm{CO} / \mathrm{O}_{2}$ (25:1:5). Spectra were normalized to the intensity at $990 \mathrm{~cm}^{-1}$. Top) Expanded view of the peroxide range. Bottom) Difference spectra for successive conditions.

This behavior indicates that reactive peroxide species, particularly those corresponding to the features at shifts of 847 and $881 \mathrm{~cm}^{-1}$, are consumed during CO oxidation and are replenished under oxygen flow. Other peroxide features at shifts of 789 and $870 \mathrm{~cm}^{-1}$ show the opposite behavior, which is suggestive of their stabilization upon exposure to $\mathrm{Ar} / \mathrm{CO} / \mathrm{O}_{2}$. Regarding the feature at a Raman shift of $789 \mathrm{~cm}^{-1}$, experiments in $\mathrm{Ar} / \mathrm{CO}$ feed, which lead to a strong increase in the Raman intensity at a shift of $789 \mathrm{~cm}^{-1}$, are indicative of stabilization by adsorbed CO.

To test this hypothesis, the dynamic behavior of the peroxide species was examined by time-dependent operando Raman spectroscopy during the onset of the CO oxidation reaction (see Figure 1, bottom panel). Figure 3 shows the temporal evolution of spectral changes in the peroxide range. No other dynamics in the spectra were observed. In each panel, two subsequently recorded Raman spectra are depicted together with the corresponding difference spectrum. The resulting sequence of difference spectra clearly shows that initially formed peroxide species at shifts of 847 and $881 \mathrm{~cm}^{-1}$ are continuously consumed during the reaction, whereas the species 


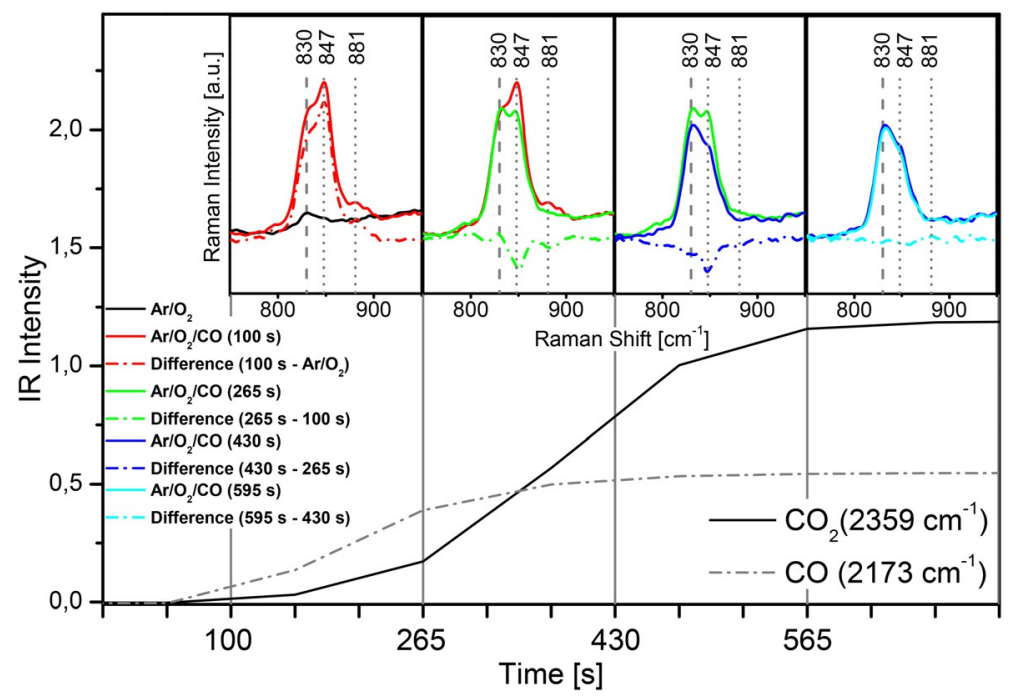

Figure 3. Operando Raman spectra of the peroxide range of $0.5 \mathrm{wt} \% \mathrm{Au} /$ ceria during the onset of the CO oxidation reaction recorded during the intervals indicated in Figure 1. Each panel shows two subsequently recorded Raman spectra together with the corresponding difference spectrum.

at the shift of $830 \mathrm{~cm}^{-1}$ does not undergo any significant changes under dynamic conditions. As the peroxide bands result from oxygen adsorption on two-electron defect sites, their consumption gives clear evidence for the participation of oxygen vacancies in $\mathrm{CO}$ oxidation over Au/ceria at room temperature.

To gain insight into the nature of the peroxide features, operando Raman spectra were recorded during CO oxidation over bare ceria (Figure S3). In the peroxide range, spectra are dominated by the feature at a Raman shift of $831 \mathrm{~cm}^{-1}$ with a minor contribution from the feature at a shift of $847 \mathrm{~cm}^{-1}$. In contrast to Au/ceria, the band at a shift of $847 \mathrm{~cm}^{-1}$ did not show any dynamics in the case of bare ceria. From this behavior we conclude that the peroxide species giving rise to the feature at a shift of $881 \mathrm{~cm}^{-1}$, which is only observed in the presence of gold, are located directly at the gold-ceria interface, whereas peroxide species related to the feature at a shift of $847 \mathrm{~cm}^{-1}$ may be associated with ceria defects (e.g., in the vicinity of the gold-ceria interface).

On the basis of our findings we propose the following processes to take place during room-temperature $\mathrm{CO}$ oxidation over Au/ceria (see Scheme 1):

1) Gas-phase oxygen molecules adsorb onto two-electron defect sites at the gold-ceria interface and are consumed by reaction with adsorbed $\mathrm{CO}$

2) Lattice oxygen is consumed by reaction with adsorbed $\mathrm{CO}$

3) Oxygen vacancies are replenished by gas-phase oxygen

The proposed processes are consistent with theoretical results for the CO oxidation mechanism on ceria-supported gold nanoparticles. ${ }^{[14]}$ According to this DFT study, oxygen vacancies near the gold-ceria interface are suggested as anchoring sites for $\mathrm{O}_{2}$ that subsequently react with $\mathrm{Au}-\mathrm{CO}$ to form $\mathrm{CO}_{2}$

\section{(2) Lattice- $\mathrm{O}^{2-} \bigcirc \mathrm{O}^{2-}$-Vacancy (ै) $^{\mathrm{T}^{2}} \mathrm{O}_{2}{ }^{2-}$-Defect $\quad \begin{aligned} & \text { Perimeter } \\ & \text { /Interface }\end{aligned}$}
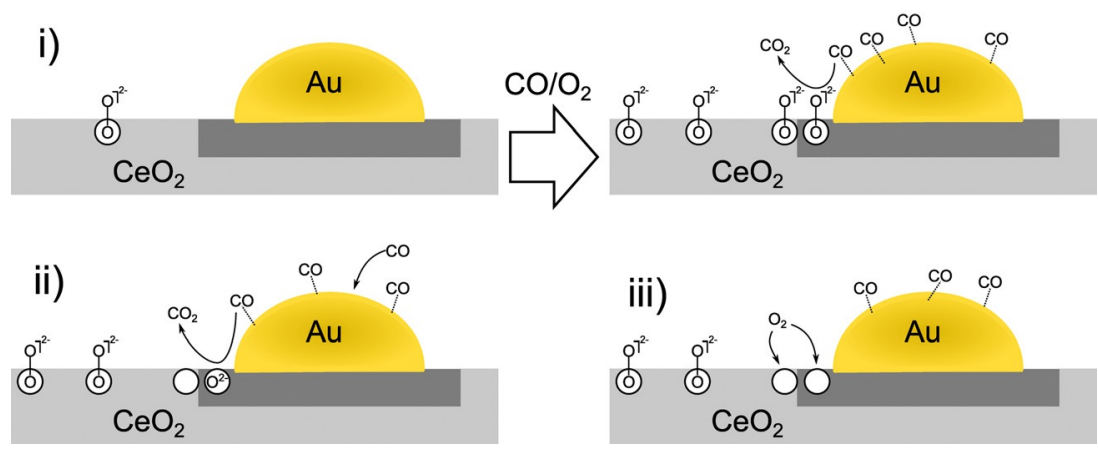

Scheme 1. Proposed processes during room-temperature CO oxidation over Au/ceria. 
[second half of the Mars-van Krevelen (MvK) mechanism]. Such oxygen-vacancy-mediated $\mathrm{O}_{2}$ activation significantly reduces the barrier for oxygen activation. During the course of the reaction, the second oxygen atom heals the vacancy. In agreement with the first half of the MvK mechanism, lattice oxygen is then consumed in a reaction with $\mathrm{CO}$, which again leads to the formation of oxygen vacancies. Considering the activation barrier for the removal of lattice oxygen, the formed $\mathrm{CO}_{2}$ is expected to stay on the surface, for example, as carbonate species (see above). The formed oxygen vacancies can be replenished by diffusion of peroxide species from the vicinity of the gold-ceria interface perimeter (species at a Raman shift of $847 \mathrm{~cm}^{-1}$ ) to the gold-ceria interface perimeter (species at a Raman shift of $881 \mathrm{~cm}^{-1}$ ). The simultaneous disappearance of both peroxide features indicates fast diffusion of peroxide species (relative to our temporal resolution). The higher intensity of the feature at a shift of $847 \mathrm{~cm}^{-1}$ originates from the fact that there is a higher abundance of oxygen vacancy sites in the vicinity than at the interface. Notably, other proposed CO oxidation mechanisms such as $\mathrm{CO}$ oxidation by co-adsorbed $\mathrm{O}_{2}$ (Langmuir-Hinshelwood mechanism) or $\mathrm{CO}$ oxidation by $\mathrm{O}_{2}$ bound to $\mathrm{Au}-\mathrm{Ce}^{3+}$ sites are not excluded by our results. Previous studies have also demonstrated the importance of oxygen vacancies in $\mathrm{CO}$ oxidation for other ceria-based catalysts. ${ }^{[2,21]}$ In the case of ceria-supported platinum catalysts, active $\mathrm{Ce}^{3+}$ species were detected during reaction by using resonant $\mathrm{X}$-ray emission spectroscopy. ${ }^{[20]}$ Doping ceria with praseodymium increases the concentration of oxygen vacancies, which in turn was shown to enhance the reactivity. ${ }^{[21]}$

Summarizing, by using operando Raman spectroscopy we provided direct spectroscopic evidence for the participation of oxygen vacancies in $\mathrm{CO}$ oxidation over ceria-supported gold catalysts at room temperature. Our results underline the crucial role of the support material for detailed understanding of the mode of operation of supported gold catalysts.

\section{Experimental Section}

The nanocrystalline ceria support was prepared by decomposition of cerium nitrate at $600^{\circ} \mathrm{C}$. Gold was loaded onto ceria by deposition precipitation of $\mathrm{HAuCl}_{4}$ by using $\mathrm{NaOH}$, as described elsewhere. ${ }^{[5]}$ The catalyst was dried overnight at $110^{\circ} \mathrm{C}$ and subsequently calcined for $12 \mathrm{~h}$ at $500^{\circ} \mathrm{C}$. The total Au content of the final catalyst was determined to be $0.5 \mathrm{wt} \%$ by using chemical analysis. The $\mathrm{Au} /$ ceria catalyst exhibited a BET surface area of $56 \mathrm{~m}^{2} \mathrm{~g}^{-1}$ and showed $<1 \%$ variation due to reaction. Transmission electron microscopy (TEM) showed the presence of $A u$ particles $3 \mathrm{~nm}$ in size. Analysis of the X-ray photoelectron spectra revealed that the majority of the gold particles were in a metallic state $\left(\mathrm{Au}^{0}\right)$ and only approximately $6 \%$ were in an oxidized state $\left(\mathrm{Au}^{+}\right)$(Figure S1).

For Raman experiments, a frequency-doubled Nd:YAG laser (Cobolt $\mathrm{AB}$ ) operated at $\lambda=532 \mathrm{~nm}$ and a transmission spectrometer (Kaiser Optical, HL5R) equipped with a charge coupled device detector (Andor) were employed. The resolution of this instrument was $5 \mathrm{~cm}^{-1}$; however, the wavelength reproducibility was better than $0.5 \mathrm{~cm}^{-1}$. The laser was operated at a power level of $2 \mathrm{~mW}$, as measured with a power meter (Ophir) at the position of the sample. Raman spectra were collected in a $180^{\circ}$ backscattering geometry by using a fiber probe (Kaiser Optical, IO-12L-VS). Operando Raman experiments were performed by using a setup described in detail elsewhere, ${ }^{[16]}$ which was extended by a FTIR spectrometer (Bruker Vertex 70, DLaTGS detector) to analyze the outlet gases of the in situ cell. The $\mathrm{CO}$ oxidation activity was monitored on the basis of the $\mathrm{CO}$ and $\mathrm{CO}_{2}$ gas-phase signals at $\tilde{v}=2173$ and $2369 \mathrm{~cm}^{-1}$, respectively.

\section{Acknowledgements}

The authors thank Christian Schilling for help with the activity measurements, Xing Huang (Fritz-Haber-Institut) and Marc Willinger (Fritz-Haber-Institut) for performing TEM experiments, as well as Karl Kopp for technical support.

Keywords: ceria $\cdot$ gold $\cdot$ nanoparticles $\cdot$ oxidation - Raman spectroscopy

[1] M. Haruta, T. Kobayashi, H. Sano, N. Yamada, Chem. Lett. 1987, 16, 405 408.

[2] M. Valden, X. Lai, D. W. Goodman, Science 1998, 281, 1647-1650.

[3] Q. Fu, H. Saltsburg, M. Flytzani-Stephanopoulos, Science 2003, 301, 935-938.

[4] X. W. Xie, Y. Li, Z. Q. Liu, M. Haruta, W. J. Shen, Nature 2009, 458, 746 749.

[5] J. Fonseca, S. Royer, N. D. Duprez, F. Epron, Appl. Catal. B 2012, 128, 10 20.

[6] G. Hutchings in Nanostructured Catalysts: Selective Oxidations, Vol. 8 (Eds.: C. Hess, R. Schlögl), RSC Publishing, Cambridge, 2011, pp. 193 213.

[7] M. Haruta, CATTECH 2002, 6, 102-105.

[8] I. X. Green, W. J. Tang, M. Neurock, J. T. Yates, Science 2011, 333, 736 739.

[9] T. Takei, T. Akita, I. Nakamura, T. Fujitani, M. Okumura, R. Okzaki, J. Huang, T. Ishida, M. Haruta in Advances in Catalysis, Vol. 55 (Eds.: B. C. Gates, F. C. Jentoft), Elsevier, Amsterdam, 2012, pp. 1-124.

[10] D. Widmann, R. J. Behm, Angew. Chem. Int. Ed. 2011, 50, 10241 -10245; Angew. Chem. 2011, 123, 10424-10428.

[11] M. M. Schubert, S. Hackenberg, A. C. van Veen, M. Muhler, V. Plzak, R. J. Behm, J. Catal. 2001, 197, 113-122.

[12] Y. Lee, G. He, A. J. Akey, R. Si, M. Flytzani-Stephanopoulos, I. P. Herman, J. Am. Chem. Soc. 2011, 133, 12952-12955.

[13] J. Guzman, S. Carrettin, A. Corma, J. Am. Chem. Soc. 2005, 127, $3286-$ 3287.

[14] H. Y. Kim, H. M. Lee, G. Henkelman, J. Am. Chem. Soc. 2012, 134, $1560-$ 1570.

[15] S. Zhang, X.-S. Li, B. Chen, X. Zhu, C. Shi, A.-M. Zhu, ACS Catal. 2014, 4, $3481-3489$.

[16] C. T. Nottbohm, C. Hess, Catal. Commun. 2012, 22, 39-42.

[17] W. H. Weber, K. C. Hass, J. R. McBride, Phys. Rev. B 1993, 48, 178-185.

[18] V. V. Pushkarev, V. I. Kovalchuk, J. L. d'Itri, J. Phys. Chem. B 2004, 108, $5341-5348$.

[19] Z. Wu, M. Li, J. Howe, H. M. Meyer, S. H. Overbury, Langmuir 2010, 26, $16595-16606$.

[20] R. Kopelent, J. A. van Bokhoven, J. Szlachetko, J. Edebeli, C. Paun, M. Nachtegaal, O. V. Safonova, Angew. Chem. Int. Ed. 2015, 54, 8728-8731; Angew. Chem. 2015, 127, 8852-8855.

[21] Z.-Y. Pu, X.-S. Liu, A.-P. Jia, Y.-L. Xie, J.-Q. Lu, M.-F. Luo, J. Phys. Chem. C 2008, 112, 15045-15051.

Received: October 15, 2015

Published online on December 17, 2015 